Virginia Commonwealth University vCU Scholars Compass

2018

\title{
Electrokinetic flow of aqueous electrolyte in amorphous silica nanotubes
}

Chris D. Daub

NTNU, TRondheim

Natalie Cann

Queens University, Ontario

D. Bratko

Virginia Commonwealth University, dbratko@vcu.edu

Alenka Luzar

Virginia Commonwealth University

Follow this and additional works at: https://scholarscompass.vcu.edu/chem_pubs

Part of the Chemistry Commons

(C) the Owner Societies 2018

\section{Downloaded from}

https://scholarscompass.vcu.edu/chem_pubs/94

This Article is brought to you for free and open access by the Dept. of Chemistry at VCU Scholars Compass. It has been accepted for inclusion in Chemistry Publications by an authorized administrator of VCU Scholars Compass. For more information, please contact libcompass@vcu.edu. 


\section{Journal Name}

\section{ARTICLE TYPE}

Cite this: DOI: $10.1039 / x x x x x x x x x x$

\section{Electrokinetic flow of aqueous electrolyte in amor- phous silica nanotubes}

\author{
Christopher D. Daub, ${ }^{a}$ Natalie M. Cann, ${ }^{b}$, D. Bratko ${ }^{c}$ and Alenka Luzar ${ }^{* c}$
}

Received Date

Accepted Date

DOI: 10.1039/xxxxxxxxxx

www.rsc.org/journalname

\section{Introduction}

As concern increases regarding the long-term viability of electric power generation from fossil fuels or nuclear fission, searches for alternative methods of electricity generation remains a popular topic for scientific study. Many methods which seek to take advantage of charge separation in aqueous ionic solutions have already been proposed. One example is the exploitation of a salinity gradient where fresh water enters the ocean 12 .

The structure of water and aqueous solutions in hydrophilic confinements of interest in energy applications has been a popular subject for molecular dynamics simulations for a long time. While we cannot give an exhaustive review, some relevant examples include the work of Leung and others on salt water in model silica pores ${ }^{314}$, as well as the studies of Argyris et al. ${ }^{5}$ and Videla et al. ${ }^{6}$. Ho et al. considered the effect of varying silica protonation states on the structure of the confined aqueous solution ${ }^{7}$. The work of Bourg and Steefel stands out in particular for their consideration of amorphous rather than crystalline silica, although they studied only pure water rather than a solution ${ }^{8}$.

Electro-osmotic flow (EOF), where an electric field is applied along the axis of the pore, has been studied in several previous works. We mention in particular the works of Moshfegh and Jabbarzadeh who studied EOF of salt water in a planar crys-

\footnotetext{
a Department of Chemistry, University of Helsinki, Helsinki, Finland FI-00014.

${ }^{b}$ Department of Chemistry, Queen's University, 90 Bader Lane, Kingston, Ontario, Canada K7L $3 N 6$

${ }^{c}$ Department of Chemistry, Virginia Commonwealth University, Richmond, VA, 232842006, USA. Tel: +1 (804) 828-3367; E-mail:aluzar@vcu.edu
}

talline model nanopore using dissipative particle dynamics 9 , and Hartkamp et al. who studied EOF of salt water in an amorphous silica nanopore using fixed-charge rigid models for water and the ions 10 . Molecular dynamics studies of fluids in nanopores which include the effect of pressure-driven flow are more rare. Lorenz et al. studied salt water under both pressure-driven flow and EOF in a channel defined by two planar crystalline silica surfaces 11 , and this work was pursued further by Haria and Lorenz ${ }^{12 \mid 13}$. Another example is the work of Zambrano et al.14 where an aqueous electrolyte film in contact with silica is examined. Botan et al. studied both EOF and pressure-driven flow of electrolyte in mineral pores $1 \frac{15}{1}$. An excellent general review of simulations of electrokinetic effects is that of Rotenberg and Pagonabarraga ${ }^{16}$. Our study concerns the electrokinetic effect in comparatively narrower pores that should enhance the overlap between the liquid velocity and ion density profiles, maximizing the difference between the water-diven fluxes of cations and anions dragged along due to their friction with water.

What sets our study apart from previous published work on aqueous salt solutions in pores is a combination of advances. First, we consider a more realistic silica pore composed of amorphous silica instead of crystalline silica. Second, in addition to fixed charge force fields ${ }^{17118}$, which we have previously used in Grand Canonical Monte Carlo simulations of the pore/salt solution partitioning 1920 , we also examine polarizable models using Drude oscillators ${ }^{21 / 22}$ to allow internal charge redistribution of water molecules and ions. Explicit polarizability may be important for describing the interfacial behaviour of the fluid more ac- 
curately, in particular the surface preference of large anions such as $\mathrm{Cl}^{-}$, even though more recent suggestions indicate that polarizability alone is not responsible for the surface preference 2324 . To the best of our knowledge our study is the first attempt to include both of these two factors in a simulation of pressure-induced flow of an ionic aqueous solution in a nanopore. As we will describe, our results differ in important ways from previous work, demonstrating the importance of considering more realistic models for both the fluid and the pore in the experimental design of devices that exploit electrokinetic effects for desalination or power generation $25[26$.

\section{Simulation methods}

We perform steady-state nonequilibrium molecular dynamics (MD) simulations of aqueous solutions of sodium chloride flowing through a silica nanopore. The importance of accurately capturing the behaviour of the ions near the silica interface motivated our choice to use the Drude oscillator based polarizable models of Lamoureux et al.22 27|28, namely the Negative Drude Particle (NDP) model for water in combination with the associated Drude ion models developed for use with the polarizable water model 29 . We have previously used these models to good effect to simulate small clusters of water and methanol containing ions 30 . We also compare these results with more standard fixed charge models for aqueous solutions, namely the SPC/E model for water 17 and the ion models of Joung and Cheatham (JC) optimized for use with SPC/E water 18 . In addition some simulations were run with a combination of the JC non-polarizable ion models and the NDP water model as a test of the different influences of the model components on the electrokinetic behaviour.

There are many available models for MD simulation of amorphous silica, including $a b$ initio methods which can correctly describe the hydrogenation of $\mathrm{SiO}$ groups at the liquid-solid interface ${ }^{3}$. For computational simplicity and efficiency we have instead taken the approach of Bourg and Steeffel ${ }^{8}$ to represent an amorphous silica nanochannel. Briefly, bulk silica is modelled using the CLAYFF potential ${ }^{31}$ which is known to produce an amorphous silica density and interatomic distributions that are in good agreement with measured values ${ }^{8}$. Starting with a configuration of crystalline silica, with bond lengths stretched so that the density matches that of amorphous silica, an NVT simulation is run at an elevated temperature of $5000 \mathrm{~K}$, followed by gradually cooling down to room temperature to produce an amorphous silica configuration.

Next, a pore aligned with the z-axis of the cell, with nominal radius of $r_{\mathrm{p}}$, is carved out from the bulk silica in a multi-step process. First, with $r_{\mathrm{xy}}=\sqrt{x^{2}+y^{2}}$ giving the distance from the pore center, all Si atoms with $r_{\mathrm{xy}}<r_{\mathrm{p}}$ are removed while $\mathrm{Si}$ atoms with $r_{\mathrm{p}} \leq r_{\mathrm{xy}}<r_{\mathrm{p}}+11 \AA$, are retained. Second, any oxygen atoms that are not within $2.3 \AA$ of at least one Si atom are also removed. Finally, all oxygen atoms which have only one Si neighbour are considered as candidates for hydrogenation. To ensure overall charge neutrality, a small number of these candidates remain dehydrogenated. The resulting surface density of silanol groups is $\sim 6.6 \mathrm{~nm}^{-2}$, in line with an earlier modeling study ${ }^{8}$ and close to the experimental estimate of $\sim 4.9 \mathrm{~nm}^{-2}$ at neutral $\mathrm{pH}^{32}$.
We also studied partially dehydrogenated surfaces, which would be a more realistic depiction of the surface at higher $\mathrm{pH}$. Preceding studies present several models for silica surfaces, differing in how the charge of the deprotonated oxygen and its neighbours are adjusted to account for the loss of each positively charged hydrogen. 10,13/15/33 37. Our approach to deprotonation involved removal of some of the pore hydrogens, along with altering the charge of the deprotonated $\mathrm{O}$ atom from $-0.95 \mathrm{e}$ to $-1.05 \mathrm{e}$, matching the rest of the non-protonated silica $\mathrm{O}$ atoms and giving a total charge difference of -0.525 e per removed $\mathrm{H}$. We balanced the loss in positive charge due to removal of each $\mathrm{H}$ by adding roughly half as many additional $\mathrm{Na}^{+}$ions. In the system with 6 additional $\mathrm{Na}^{+}, 12 \mathrm{H}$ were removed and the extra system charge of -0.03 e was countered by slightly increasing the charge of each remaining $\mathrm{H}$. The system with 42 additional $\mathrm{Na}^{+}$ had $80 \mathrm{H}$ removed to balance the system exactly. This approach minimizes the required adjustment of the deprotonated oxygen charge, and is similar to that used in Refs. 34 and 35.

The pore radius $r_{\mathrm{p}}=10 \AA$ and four separate independent starting silica configurations were generated. The length of the pore is $40.56 \AA$ for all simulations. It is worth noting that the pore radius is only roughly described by $r_{\mathrm{p}}$ since $\mathrm{O}, \mathrm{O}^{-}$, and $\mathrm{OH}$ groups extend towards the pore center while the amorphous silica naturally has empty "pockets" that allow solvent and ions to penetrate the solid beyond $r_{\mathrm{p}}$.

At this point, after generation of the empty silica pore, water was added to the interior of the pore. In order to minimize the system size, as well as to allow us to form an infinitely long pore, we wished to avoid including the open reservoir outside the pore used in previous work ${ }^{8}$. Instead, separate $N V T$ simulations of bulk water were done at $298 \mathrm{~K}$. These provided bulk water configurations to form an initial representation of water in the pore: all water molecules except those in the interior of the pore which did not significantly overlap with the silica were removed. During subsequent equilibration of the water in the silica pore, we found that water molecules slowly penetrated the silica cavities, so that the fluid density became significantly reduced. We mitigated this effect by generating our bulk water configurations at elevated densities. Using the initial density of $1.6 \mathrm{~g} / \mathrm{cm}^{3}$ before filling the pore, the density of the fluid in the center of the pore after equilibration was near $1 \mathrm{~g} / \mathrm{cm}^{3}$. Finally, we randomly selected water molecules to convert into ions according to the desired concentration and degree of surface hydrogenation. We were careful to avoid initially placing ions in close proximity to surface cavities, to avoid ions artificially adsorbing too strongly. Representative snapshots of the equilibrated pores are shown in Figure 1 .

Several methods have been proposed for simulating pressureinduced fluid flow. We have chosen the simplest option, where a gravity-like force $F_{z}=A_{g} \times m_{i}$ is applied to every atom in the fluid. Although in some ways this method is less realistic, it has been shown to give results which agree well with more complex methods 11 . In particular, it causes the fluid flow identical to that due to the equivalent pressure gradient $\nabla P_{z}=-\rho A_{g}$, which is harder to implement, especially when one wants to make use of periodic boundaries. Above, $\rho$ is the fluid density in the pore. We used 

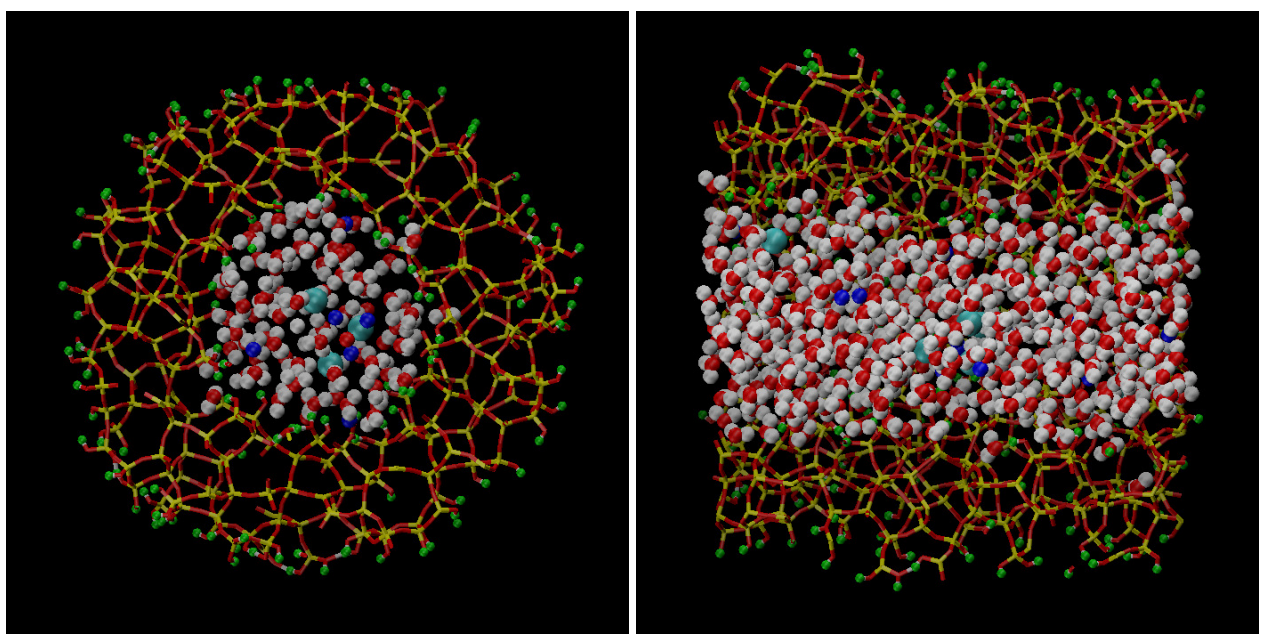

Fig. 1 Snapshots of the equilibrated pore with $r_{p}=10 \AA$ filled with water and 6 ion pairs $\left(\sim 1 \mathrm{~mol} \mathrm{dm}^{-3}\right.$ solution). Si atoms are shown in yellow, O in red, Silanol $\mathrm{H}$ in green, water $\mathrm{H}$ in white, $\mathrm{Na}^{+}$in blue and $\mathrm{Cl}^{-}$in cyan. Relative atomic sizes are not accurately depicted. (Left) Front view showing only $10 \AA ̊$ of the pore and fluid atoms. (Right) Side view with the front half of the pore atoms removed for clarity.

three different values of the acceleration $A_{g}$ in this work. The lowest value is $A_{1}=0.00368 \mathrm{kcal} \AA^{-1} \mathrm{~g}^{-1}$, with other simulations being run at values two and three times higher. This value of $A_{g}$ is equivalent to the pressure gradient $\nabla P_{z} \simeq 1.5 \times 10^{12} \mathrm{~atm} \mathrm{~m}^{-1}$. These are very large pressure gradients, likely far above what could be feasible experimentally, but are necessary in our case to obtain sufficiently high simulated flow rates in very narrow pores. Pressure gradients of identical magnitudes $\left(0.8-2.4 \times 10^{12} \mathrm{~atm}\right.$ $\mathrm{m}^{-1}$ ) were used to study flow through nanoporous graphene 38 , whereas two orders of magnitude lower gradients sufficed to simulate flow in a larger planar nanopore made of amorphous silica ${ }^{111}$. Induced flow was also studied by Wang et al. 39 in a narrow carbon nanotube using pressure gradients $\nabla P_{z}$ of up to $\simeq 1.35 \times 10^{10} \mathrm{~atm} \mathrm{~m}^{-1}$. The smoothness and hydrophobicity of the nanotube somewhat mitigated the need for extremely large pressures in this case.

Rigorous nonequilibrium simulations account for thermal gradients associated with the dissipative flow through the pore by various applications of thermostats to the pore wall atoms only 40,43 , with the fluid's resulting temperature determined via heat flow from the walls. As we use thermostats to control the temperature of both the pore and the fluid directly, our results correspond to the high thermal conductance limit with suppressed temperature gradients, which renders our results scalable to significantly weaker pressure (and thermal) gradients than the larger gradients we have been required to apply for the sake of computational advantage only.

In our early tests, we found that if a single thermostat was applied to the entire system, even though the average temperature would be maintained by the thermostat, over the course of a long simulation the temperatures of the wall and the fluid would become significantly different. The system was hence thermostatted by two separate Nose-Hoover thermostats ${ }^{44}$, both with time constant $100 \mathrm{fs}$, one of which acted on the pore walls and the other on the solution. Both thermostats were set to maintain a temperature of $298 \mathrm{~K}$ and generally held temperature fluctuations across the system below 2-3 degrees.

We have also studied the application of an external electric field to the system under four sets of conditions; with electrolyte at two different concentrations flowing through an electrically neutral silica pore, and two electrolytes flowing through a partially deprotonated pore. These conditions provide a means to examine the effect of $\mathrm{pH}$ - via channel deprotonation - and electrolyte concentration on electrokinetic flow. This will also allow us to estimate the relation between the electric current generated and the applied mechanical power, since several combinations of electric and gravitational fields are examined, in order to assess the theoretical power generation efficiency of our systems.

In our simulations, consideration of boundary conditions is crucial. To avoid interactions between pores, the full simulation cell consists of the silica pore aligned along $\mathrm{z}$, the enclosed electrolyte fluid, and empty space on the sides of the silica. Overall, the orthorhombic simulation cell $L_{x, y} \times L_{x, y} \times L_{z}$ has dimensions $63.68 \times 63.68 \times 40.56 \AA$. We apply 3D periodic boundary conditions, but with the appropriate Ewald correction terms for a system infinite in only one dimension, along the pore length, with vacuum boundaries in the other two dimensions $45 / 46$. This allows us to use the standard 3D Ewald sum to compute the Coulombic interactions in our pseudo-1D system with a high degree of accuracy. We added the usual fictitious extra empty space $d_{\text {slab }}=3 L_{x, y}$ in the Ewald sum to ensure negligible electrostatic interactions with the periodic images in the $x$ and $y$ dimensions ${ }^{46}$.

\section{Results and Discussion}

As explained in the previous section, we studied a variety of different systems, including distinct atomic/molecular force fields, different pore widths, degrees of surface hydrogenation, ionic concentrations, applied flow force, and electric fields. In Tables 1 and 2 we summarize all of the different systems we have examined. The ion concentrations are approximate because the pore surface is rough and not well defined.

In the remainder of this section, simulations with the NDP wa- 
ter and polarizable ions models will be identified by $P O L_{A L L}$. $N O P O L_{A L L}$ identifies simulations of the non-polarizable SPC/E water and the associated Joung and Cheatam ions. Finally, when the NDP water model is paired with the non-polarizable JoungCheatam ions, the simulations are identified by $P O L_{H 2 O}$.

\subsection{Velocity profiles}

\subsubsection{Induced flow simulations}

In the left part of Figure 2 we show profiles of the average velocity of water molecules in the simulations under flow with both $P O L_{A L L}$ and NOPOL $L_{A L L}$ models. Results are shown for the systems with 6 sodium and chloride ions; results for pure water and other ion concentrations were not qualitatively different.

The Hagen-Poiseuille law for pressure-driven flow in a cylindrical channel with pore radius $r_{\mathrm{p}}$ can be expressed as 47

$$
v_{z}\left(r_{x y}\right)=-\frac{\Delta P}{4 \eta L_{z}}\left[r_{\mathrm{p}}^{2}+2 b r_{\mathrm{p}}-r_{x y}^{2}\right]
$$

where $\Delta P$ represents the pressure difference induced in a pore of length $L_{z}, \eta$ is the fluid viscosity, $b$ is the slip length and $r_{x y}$ is the radial distance from the center of the channel. Equation 1 is exact for an incompressible fluid of constant viscosity travelling through a perfectly cylindrical channel with length far greater than the pore radius. In contrast to results in smooth carbon nanotubes, which have large positive slip lengths $b$, we see that the velocity for amorphous silica vanishes some distance away from the interface, consistent with a negative slip length, and reflecting the surface roughness and the strong surface-fluid interactions. We fit the velocity profiles with a two-parameter quadratic function consistent with Equation 1

$$
v_{z}\left(r_{x y}\right)=a_{0}+a_{1} r_{x y}^{2}
$$

which works quite well in all cases, as shown in the left side of Figure 2 In Table 3 we show the average results for these fits over the four independent initial pore configurations. We also show estimates of the slip length $b$ obtained from the fit parameters and the pore diameter $r_{\mathrm{p}}=10 \AA$. The slip length is $\sim-2$ to $-2.5 \AA$ slightly longer with the non-polarizable SPC/E model than the polarizable NDP model. The slip length is also slightly reduced as the induced force $F_{z}$ is increased. This small negative slip length, consistent with the presence of approximately one molecular layer of mostly immobile water and ions, is consistent with previous simulations showing a slip length $b=-3 \AA$ for water flowing in a hydrophilic diamond nanopore functionalized with $\mathrm{OH}$ groups 48 .

An alternative interpretation of Equation 1 is to view the fit of Equation 2 as defining an effective, field-dependent pore radius. In support of this interpretation, the water velocities in Figure 2 are near zero from $r_{x y}>8.0 \AA$ indicative of a zero-slip boundary, $b=0$, located at a distance of roughly $r_{x y} \sim 8.0 \AA$. Table 3 lists values of this effective pore radius $r_{\mathrm{p}}^{\text {eff }}$ where the fitted flow velocity is zero in the last column. The effective radius $r_{\mathrm{p}}^{\text {eff }}$ increases with the field, as expected, since molecules near the wall are more likely to be dislodged under a stronger field, and $r_{\mathrm{p}}^{\text {eff }}$ is larger for the $P O L_{A L L}$ fluid indicating that polarizability increases mobility near the surface. This can also be seen in the plot of $v_{z}\left(r_{x y}\right)$ in Figure 2 where the Drude model water velocities near the interface are higher than for SPC/E water.

Although the data for each individual pore and induced flow rate does agree rather well with the Hagen-Poiseuille prediction, a significant deviation is observed in the scaling of the constant fit parameter $a_{0}$, or in other words the maximum flow velocity $v_{z, \text { max }}$ in the middle of the pore, which would be expected to vary linearly with the induced flow force $A_{g} \propto \Delta P$ (assuming constant viscosity). In the right side of Figure 2 we show the plot of $v_{z, \max }$ as a function of $A_{g}$, and a fit to a power law with an exponent of 1.35. Possible explanations of the nonlinearity include a decrease in the effective viscosity of our system below the bulk predictions for the SWM4-NDP water model ${ }^{28}(\eta=0.7 \mathrm{cp})$ and SPC/E model ${ }^{49}(\eta=0.729 \mathrm{cp})$, or the breakdown of the Hagen-Poiseuille law due to the large pressure gradients we exert. A similar flow enhancement has previously been reported in MD simulations in nanosized carbon nanotubes, however, the partial slip observed

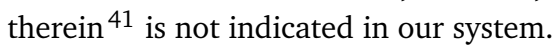

\subsubsection{Electro-osmotic flow simulations}

In Figure 3we show the velocity profiles of different species under a DC electric field $E=0.5 \mathrm{~V} / \mathrm{nm}$ applied parallel to the pore axis for the $N O P O L_{A L L}$ simulations. Data for the polarizable model simulations are not shown since the long runs required to resolve these velocity profiles were not possible in that case. In contrast to the previous case of pressure-induced flow, here the electric field drives very different flow profiles for the different species. As one might expect, $\mathrm{Cl}^{-}$anions flow against the field direction. Due to the overall greater flux of $\mathrm{Cl}^{-}$versus $\mathrm{Na}^{+}$, we also observe a weak electro-osmotic effect whereby the water molecules on average are dragged along with the $\mathrm{Cl}^{-}$in the negative direction. Our EOF results show a qualitative resemblance with recent MD simulations of electro-osmotic flow between montmorillonite plates 15 . The difference between the narrow-cylinder pore geometry used in our work and about three times wider planar pores in Ref. 15, as well as the considerably larger electric fields they applied, can explain considerably higher electro-osmotic mobility observed in the latter system.

Figure 3 also shows the velocity profiles in the induced flow simulations. Unlike the simulations of electro-osmotic flow, here we see that all of the different species have a similar velocity profile, as the free ions are carried by the flow of water. Another interesting difference to note in the EOF simulations is that, unlike in the case of the pressure-induced flow simulations where the velocity profile fit well to the classical Poiseuille law prediction of a parabolic profile, in this case the classical law is not obeyed. While the classical law would predict flow obeying the Smoluchowski equation, i.e. a constant plateau velocity across the pore for each species 11 , here we can see no point where the velocity histogram becomes flat. We attribute this difference to the small size of the pore, which is on the order of the typical Debye length of aqueous systems (ca. 1-2 nm). 
Table 1 Summary of all systems studied in the presence of a gravity-like field, but without electric field. The degree of pore deprotonation is specified in the first column. The electrolyte characteristics are given in Columns 2 and 3 with the number of each ion provided along with the corresponding approximate concentration. Simulation specifics are provided in the final columns: Column 4 gives the strength of the gravity-like forces examined; the number of independent runs, per force strength, is given in Column 5; and the final column gives the total simulation time, for each force strength listed

\begin{tabular}{|c|c|c|c|c|c|}
\hline Pore type & $N_{N a^{+}},\left[N a^{+}\right]$ & $\mathrm{N}_{\mathrm{Cl}^{-}},\left[\mathrm{Cl}^{-}\right]$ & $\overline{A_{g} / A_{1}}$ & \# runs & time/ns \\
\hline \multicolumn{6}{|l|}{$P O L_{A L L}$} \\
\hline \multirow[t]{3}{*}{$\overline{\text { Protonated }}$} & 0 & 0 & $0,1,2,3$ & 4 & 1.625 \\
\hline & $6,[0.78 \mathrm{M}]$ & $6,[0.78 \mathrm{M}]$ & $0,1,2,3$ & 4 & 4.5 \\
\hline & $12,[1.56 \mathrm{M}]$ & $12,[1.56 \mathrm{M}]$ & $0,1,2,3$ & 3 & 3.5 \\
\hline \multirow[t]{2}{*}{ Deprotonated } & $12,[1.56 \mathrm{M}]$ & $6,[0.78 \mathrm{M}]$ & 0,2 & 2 & 3.5 \\
\hline & $48,[6.25 \mathrm{M}]$ & $6,[0.78 \mathrm{M}]$ & 0,2 & 2 & 3.5 \\
\hline \multicolumn{6}{|l|}{${ }_{N O P O L_{A L L}}$} \\
\hline \multirow[t]{2}{*}{$\overline{\text { Protonated }}$} & 0 & 0 & $0,1,2$ & 2 & 12.5 \\
\hline & $6,[0.78 \mathrm{M}]$ & $6,[0.78 \mathrm{M}]$ & $0,1,2$ & 4 & 24 \\
\hline Deprotonated & $12,[1.56 \mathrm{M}]$ & $6,[0.78 \mathrm{M}]$ & $0,1,2$ & 2 & 24 \\
\hline \multicolumn{6}{|l|}{$\mathrm{POL}_{\mathrm{H}_{2} \mathrm{O}}$} \\
\hline Protonated & $6,[0.78 \mathrm{M}]$ & $6,[0.78 \mathrm{M}]$ & 0,2 & 4 & 4.5 \\
\hline
\end{tabular}

Table 2 Summary of all systems studied, with both an applied force $F_{z}$ and an applied electric field $E_{z}$. The first column identifies whether the pore is neutral or partially deprotonated. The following two columns identify the number of each ion (the associated concentration is available in Table 1). The strength of the gravity-like and electric fields are given in Columns 3 and 4 . The final two columns identify the number of runs and total run time for each $F_{z}$ and $E_{z}$ combination.

\begin{tabular}{|c|c|c|c|c|c|}
\hline Pore type & $N_{\mathrm{Na}^{+}}, \mathrm{N}_{\mathrm{Cl}^{-}}$ & $A_{g} / A_{1}$ & $E_{z} / V \mathrm{~nm}^{-1}$ & \# of runs & run time/ns \\
\hline \multicolumn{6}{|l|}{$P O L_{A L L}$} \\
\hline \multirow[t]{4}{*}{$\overline{\text { Protonated }}$} & 6,6 & 2 & $-0.05,-0.1$ & 4 & 4 \\
\hline & & 3 & $-0.05,-0.1,-0.2$ & 4 & 4 \\
\hline & 12,12 & 2 & $-0.05,-0.1$ & 2 & 4 \\
\hline & & 3 & $-0.05,-0.1,-0.2$ & 4 & 4 \\
\hline \multirow{4}{*}{ Deprotonated } & 12,6 & 2 & $-0.05,-0.1$ & 2 & 4 \\
\hline & & 3 & $-0.05,-0.1,-0.2$ & 2 & 4 \\
\hline & 48,6 & 2 & $-0.05,-0.1$ & 2 & 4 \\
\hline & & 3 & $-0.05,-0.1,-0.2$ & 2 & 4 \\
\hline \multicolumn{6}{|l|}{$N^{\prime O P O L} L_{A L L}$} \\
\hline \multirow[t]{3}{*}{ Protonated } & 6,6 & 0 & $0.1,0.2,0.5$ & 4 & 28 \\
\hline & & 1 & $0.1,0.2,0,5$ & 4 & 28 \\
\hline & & 2 & $0.1,0.2,0.5$ & 4 & 28 \\
\hline \multirow[t]{2}{*}{ Deprotonated } & 12,6 & 1 & $0.1,0.2,0.5$ & 4 & 24 \\
\hline & & 2 & $0.1,0.2,0.5$ & 4 & 24 \\
\hline
\end{tabular}

Table 3 Fit of the velocity profiles for varying values of $A_{g}$ (with $A_{1}=0.00368 \mathrm{kcal} \AA^{-1} \mathrm{~g}^{-1}$ ) and with different water models to the quadratic model in Equation 2 and resultant slip lengths $b$. Error bounds are the standard error in the mean fit parameters and slip lengths over 4 independent trajectories from different initial configurations. The fit parameters can also be expressed as $a_{0}=-\frac{\Delta P}{4 \eta L_{z}}\left(r_{\mathrm{p}}^{2}+2 b r_{\mathrm{p}}\right)$ and $a_{1}=\frac{\Delta P}{4 \eta L_{z}}$, and the slip length $b=-a_{0} /\left(2 a_{1} r_{\mathrm{p}}\right)-r_{\mathrm{p}} / 2$

\begin{tabular}{|c|c|c|c|c|}
\hline & $a_{0} / \mathrm{m} \mathrm{s}^{-1}$ & $a_{1} / \mathrm{m} \mathrm{s}^{-1} \AA^{-2}$ & $b / \AA$ & $r_{\mathrm{p}}^{e f f} / \AA$ \\
\hline$\overline{N O P O L_{A L L}, A_{g}=A_{1}}$ & $17.9 \pm 0.2$ & $-0.346 \pm 0.005$ & $-2.40 \pm 0.03$ & $7.20 \pm 0.10$ \\
\hline$N O P O L_{A L L}, A_{g}=2 A_{1}$ & $42.1 \pm 0.6$ & $-0.797 \pm 0.013$ & $-2.35 \pm 0.02$ & $7.27 \pm 0.11$ \\
\hline$\overline{P O L_{A L L}, A_{g}=A_{1}}$ & $17.1 \pm 0.5$ & $-0.306 \pm 0.013$ & $-2.21 \pm 0.05$ & $7.48 \pm 0.26$ \\
\hline$P O L_{A L L}, A_{g}=2 A_{1}$ & $43.1 \pm 1.0$ & $-0.724 \pm 0.022$ & $-2.03 \pm 0.03$ & $7.72 \pm 0.21$ \\
\hline$P O L_{A L L}, A_{g}=3 A_{1}$ & $74.9 \pm 1.0$ & $-1.199 \pm 0.023$ & $-1.87 \pm 0.04$ & $8.04 \pm 0.26$ \\
\hline
\end{tabular}



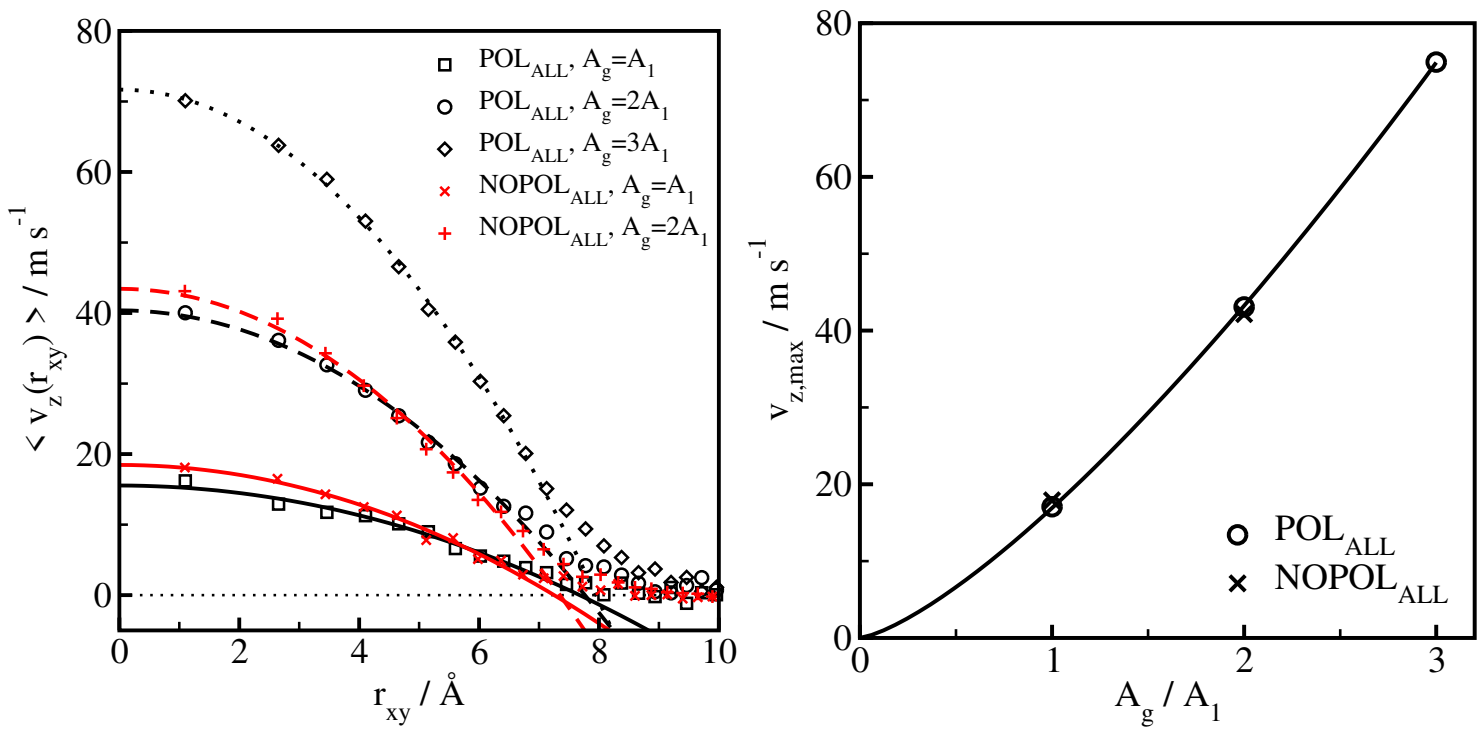

Fig. 2 (Left) The average velocity of water molecules along the pore direction at varying distances from the pore centre, and corresponding fits to the Hagen-Poiseuille law (Equation 1). Results are shown for the $r_{\mathrm{p}}=10 \AA$ silica channel in contact with water and $6 \mathrm{Na}^{+}$and $6 \mathrm{Cl}^{-}$ions. Only one trajectory is shown for each model and induced gravity field strength $A_{g}$. The fit only includes data points with $r_{x y}<7 \AA$. (Right) Plot of the maximum flow velocity $v_{z, \max }$ as a function of $A_{g}$ showing the fit to a power law $v_{z, \max } \propto A_{g}^{1.35}$.

\subsection{Density profiles}

Here and in the remainder of the paper, we will focus only on the simulations with the $r_{\mathrm{p}}=10 \AA$ pore. Although the qualitative picture in the larger $r_{\mathrm{p}}=15 \AA$ pore was similar, the increased system size and shorter simulation times made gathering enough data to make a quantitative analysis very difficult.

In Figure 4 we show the density distributions for the water, $\mathrm{Na}^{+}$, and $\mathrm{Cl}^{-}$observed in the absence of pressure-induced flow and for $A_{g}=2.0$. In contrast with the velocity histograms in the induced flow simulations, which were quite similar for the different chemical species and systems/models (cf. Figures 2 and 3), in the density histograms we see important differences. In particular, with all models we note that there is some tendency for all ions to be located near the interface, and to form comparatively long-lived $\mathrm{Na}^{+}-\mathrm{O}$ or $\mathrm{Cl}^{-}-\mathrm{HO}$ bonds regardless of whether there is pressure-driven flow. While these interactions are maintained, the ions are effectively immobilized. It is the variations in the likelihood of these interactions which will lead to differences in the ion flux that we analyze further in the following sections.

The water density profiles look rather similar to those seen in previous work on similar silica interfaces 8 [11, with a flat plateau near the center of the pore and a layer of somewhat higher density next to the hydrophilic SiOH interface. Beyond 7.0 ̊, the water density gradually drops to zero while the ions are most likely to be found at these locations near the surface. The water density in the middle of the channel is somewhat lower than that of bulk solution. The slow percolation of water into the cavities of the amorphous $\mathrm{SiOH}$ led to the density being slightly lower than we intended, however we have compared our results with test simulations with even lower water density (not shown) and obtained similar results.

Introduction of flow through the channel reduces the chances of finding the ions near the surface, but the overall probability for binding remains high. Of course, this flow-induced decrease at the surface must be compensated with an increase in the middle of the pore. In the NOPOL $L_{A L L}$ simulations, the $\mathrm{Na}^{+}$cations have a higher probability to be bound and few cations are found within $5 \AA$ of the pore center. Inclusion of polarizability reduces the overall density of bound ions. Introduction of flow reduces the probability further with the biggest impact for $\mathrm{Na}^{+}$. In contrast to the $N_{O P O L} L_{A L L}$ simulations, it is now the $\mathrm{Cl}^{-}$anions which show a higher density near the interface instead of the $\mathrm{Na}^{+}$. Overall, the two different force fields predict rather similar distributions for water and $\mathrm{Cl}^{-}$, but very large differences for $\mathrm{Na}^{+}$.

\subsection{Binding time histograms}

Analysis of the velocity of each ion in the induced flow simulations is used to determine when binding events start and end, and provide a distribution of binding times of the various ions and how they vary with our different models. The histograms in Figure 5 show that the polarizable systems (red and black bars) feature much shorter binding times than the nonpolarizable (blue, green) ones. Both ion and water force fields affect ion binding. Just replacing the SPC/E water model by the polarizable NDP one, while keeping the nonpolarizable (JC) ions (middle plot in Figure 5) eliminates most of the long-lasting binding events.

Knowledge of the binding times also gives us the possibility to determine the average fraction of the total simulation time $F_{b i n d}$ the ions are bound. Conversely, $1-F_{\text {bind }}=F_{\text {flow }}$ is the fraction of time the ions are moving due to the induced flow. The ion current for each ion is a product of the time $F_{\text {flow }}$ multiplied by the velocity $v_{\text {ion }}$ of a moving ion; if we assume that ion velocities are on average more or less the same across species, as suggested by Figure 3 then $F_{\text {flow }}$ might be used to approximately estimate the contributions of each species to the differential ionic currents analyzed more thoroughly in the next section ( $c f$. Figure 6). In Ta- 

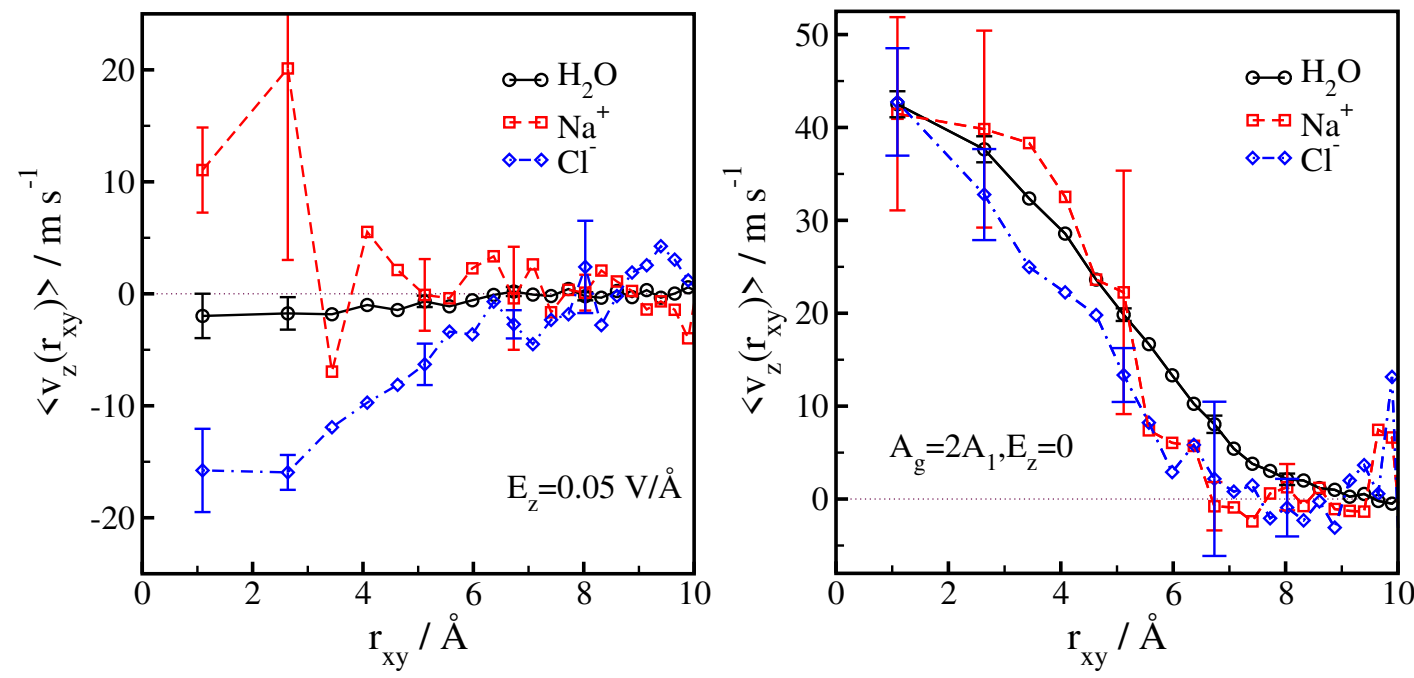

Fig. 3 Plot of the average velocity $v_{z}$ of different species under an applied DC electric field (left) and under an induced flow force (right). All data comes from the $N O P O L_{A L L}$ system with $r_{\mathrm{p}}=10 \AA$ and 6 ion pairs. Error bars shown for some representative points are one standard deviation in the data from the four independent starting configurations. Lines joining points are meant only as a guide to the eye.

Table 4 Analysis of the fraction of time $F$ that each ion is found to be flowing $\left(F_{\text {flow }}=1-F_{\text {bind }}\right)$ in each system, as well as the computation of the relative difference $I_{\text {rel }}=N \times\left(F_{\text {flow }, \mathrm{Na}^{+}}\right)-F_{\text {flow }, \mathrm{Cl}}$. Factors $N$ (listed in Figure 6 equal $\mathrm{N}_{\mathrm{Na}^{+}} / \mathrm{N}_{\mathrm{Cl}^{-}}$in these examples.

\begin{tabular}{lllll} 
System & $A_{g} / A_{1}$ & $F_{\text {flow }, \mathrm{Na}^{+}}$ & $F_{\text {flow, } \mathrm{Cl}}$ & $I_{\text {rel }}$ \\
\hline$P O L_{A L L}, 12 \mathrm{Na}^{+}+6 \mathrm{Cl}^{-}$ & 2.0 & 0.76 & 0.75 & 0.76 \\
POL $_{A L L}, 6 \mathrm{Na}^{+}+6 \mathrm{Cl}^{-}$ & 2.0 & 0.73 & 0.50 & 0.23 \\
$P^{-} O L_{A L L}, 6 \mathrm{Na}^{+}+6 \mathrm{Cl}^{-}$ & 3.0 & 0.75 & 0.46 & 0.29 \\
POL $_{\mathrm{H}_{2} O}, 6 \mathrm{Na}^{+}+6 \mathrm{Cl}^{-}$ & 2.0 & 0.64 & 0.76 & -0.11 \\
NOPOL $_{A L L}, 12 \mathrm{Na}^{+}+6 \mathrm{Cl}^{-}$ & 2.0 & 0.18 & 0.63 & -0.27 \\
NOPOL $_{A L L}, 6 \mathrm{Na}^{+}+6 \mathrm{Cl}^{-}$ & 1.0 & 0.07 & 0.28 & -0.21 \\
NOPOL $_{A L L}, 6 \mathrm{Na}^{+}+6 \mathrm{Cl}^{-}$ & 2.0 & 0.11 & 0.35 & -0.24
\end{tabular}

ble 4 we can see that this crude approximation does work rather well, and provides a simplified way to understand how the characteristics of the different systems and molecular models used lead to the different electrokinetic behaviours we observe.

Also included in Table 4 are some results for different applied flow rates $A_{g}$. These demonstrate that the fractional binding times $F_{b i n d}$ are not very sensitive to differences in the flow velocity. Multiplication of the relative difference $I_{\text {rel }}=N \times\left(F_{\text {flow }, N a^{+}}\right)-$ $F_{\text {flow }, \mathrm{Cl}^{-}}$, with $N$ a numerical factor for the relative difference in concentration of each ionic species as shown in Figure 6, by the average flow velocity determined for example from the velocity histogram, roughly agrees with the data shown by a more thorough analysis in the next section and in Figure 6.

It is evident from looking at Table 4 that both the choice of ion model as well as the water models used play a role in determining the overall electrokinetics. Although combining the nonpolarizable Joung-Cheatam ion model with the polarizable water model does cause the current polarity to reverse compared with the polarizable ion model, the very low mobility of the sodium ions in particular is only seen when using the fixed-charge water model.

As the ions move in the channel, they can enter a void in the amorphous surface and, from there, travel further in to become embedded within the silica. This is an infrequent occurrence but is evident from the non-zero densities beyond $r_{x y}=10 \AA$ in Figure 4. Once trapped in the silica, an ion will usually remain there for the remainder of the simulation. This occurs more frequently in the $N O P O L_{A L L}$ systems than in the $P O L_{A L L}$ systems.

The weaker binding propensity predicted from the polarizable force field calculations is in reasonable agreement with potentiometric experiments ${ }^{50}$ and recent MD simulations ${ }^{51}$. Specifically, the standard free energy of binding of the sodium ions $\Delta G^{o} \simeq-k_{B} T \ln \left(\rho_{\text {contact }} / \rho_{\text {free }}\right)$ estimated from the ion distributions of Ref. [51] is $\simeq-1.2 k_{B} T$, relatively close to the value $\simeq-0.9 k_{B} T$ following from our polarizable force field calculations (Figure 4) and very different from the value of $\simeq-4.0 k_{B} T$ suggested by the ion distributions (Figure 4) for the nonpolarizable force field system. Recent findings that electrometric measurements of $\mathrm{Na}^{+}$ binding to phospholipid bilayers are overestimated by simulations using non-polarizable models ${ }^{52}$, and can be corrected by the inclusion of polarizability 53 , are also consistent with a conclusion that simulations using non-polarizable force fields lead to overestimates of the degree of $\mathrm{Na}^{+}$-silica binding. Certainly we can expect some sensitivity of our results to changes in the ion models; it has been noted by Bourg et al. that the Joung-Cheatham ion parameters we have used can overestimate the entry of $\mathrm{Na}^{+}$ ions into the hexagonal cavities of the surface of mica ${ }^{54}$, and perhaps changing to the models of Dang and Chang ${ }^{55}$ would not so greatly overestimate the $\mathrm{Na}^{+}$binding. At the same time, it is clear from our simulations with the $\mathrm{POL}_{\mathrm{H}_{2} \mathrm{O}}$ system that altering the ion model alone would be unlikely to fully correct the description of the $\mathrm{Na}^{+}$binding.

\subsection{Induced current and energy-conversion efficiency anal- ysis}

Figure 6 shows the difference in the average velocity of ions, $\left(N_{\mathrm{Na}^{+}}\left\langle v_{\mathrm{Na}^{+}}\right\rangle-N_{\mathrm{Cl}^{-}}\left\langle v_{\mathrm{Cl}^{-}}\right\rangle\right) / 6 . \quad N_{\mathrm{Cl}^{-}}$is held at 6 in deprotonated 

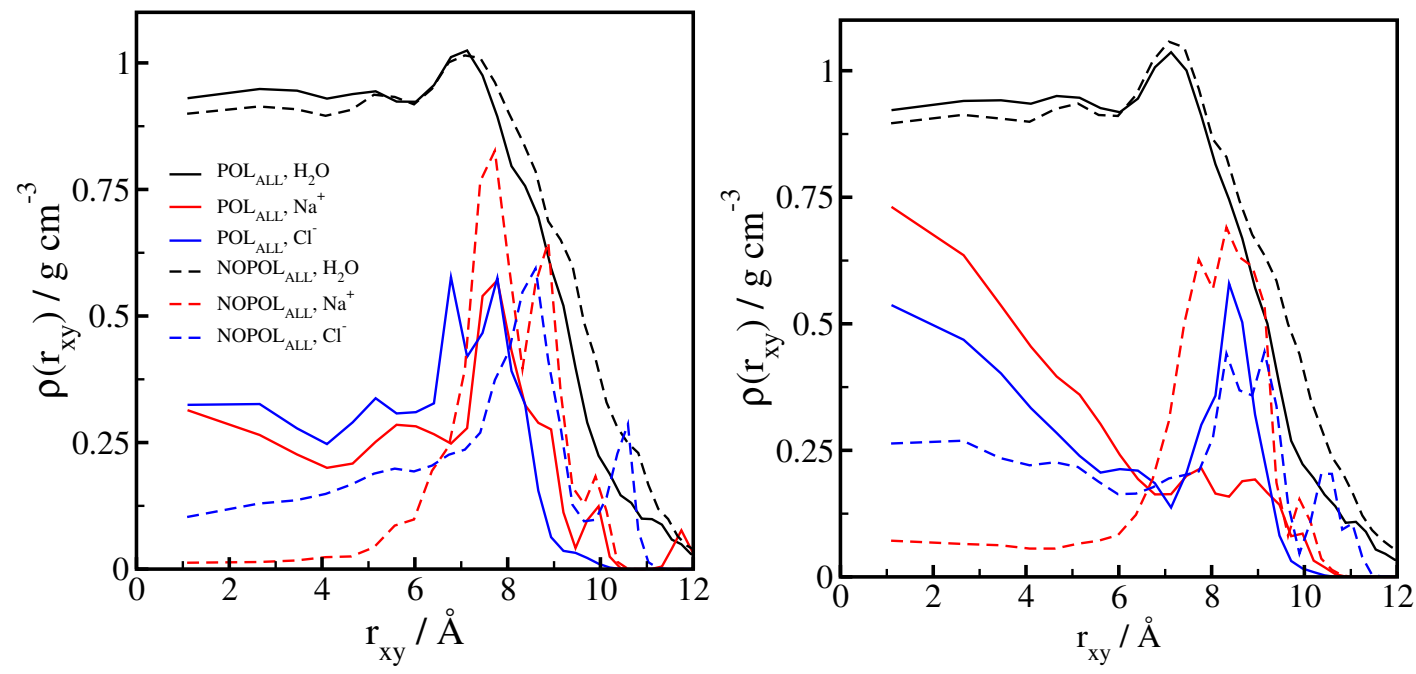

Fig. 4 The density of different species within the pore as a function of distance from the center. Results are shown for zero induced flow (left) and for a flow with $A_{g} / A_{1}=2.0$ (right) in the system with 6 ion pairs and $r_{p}=10 \AA$. The ion-wall binding is significantly weakened under the flow (right plot). Water density is in $\mathrm{g} \mathrm{cm}^{-3}$; ion densities are proportional to the number density in arbitrary units. The applied electric field is zero in all cases.

pores while it equals $N_{\mathrm{Na}^{+}}$in neutral (fully protonated) ones. It is the above differences in the average ion velocity which will translate into the generation of electric current $I=e_{o}\left(N_{N a^{+}}\left\langle v_{N a^{+}}\right\rangle-\right.$ $\left.N_{C l^{-}}\left\langle v_{C l^{-}}\right\rangle\right) / L_{z}$ in a laboratory version of our simulations.

We see that, in agreement with the findings for the density distributions shown in Figure 4 the average ionic flows due to the difference in binding propensities are significantly different for the different ions. In the fixed charge simulations, the larger likelihood of cations to be bound gives an overall negative charge current, while the polarizable model simulations instead give a positive charge current.

By running simulations with an electric field applied in a direction which counteracts the flow-induced charge flux, we can estimate the electric work and the efficiency of mechanical-toelectric energy conversion. We can also estimate the Onsager phenomenological coefficients describing the coupling between the electric field and the flow-induced charge separation. These data are shown in Tables 5 and 6.

As long as the system at a specified $\nabla P$ obeys Ohm's law (constant $\left.(d I / d E)_{\nabla P}\right)$, the optimal energy conversion can be obtained when the inner and load resistances, and concomitant voltage drops, are equal. This results in electric current $I=I_{\max } / 2$ and electric power $P_{e l}=I_{\max } E_{o} / 4$. Here $E_{o}$ represents the field strength just sufficient to reduce the electrokinetic current at given $\nabla P$ from $I_{\max }$ in the absence of applied field. The conversion efficiencies $\varepsilon=P_{e l} / P_{\text {mech }}$ are collected in Table $5 \varepsilon$ is $0.2 \%$ or less in the fully protonated $P O L_{A L L}$ systems, and rises by an approximate factor of 10 to $1-2 \%$ in the partially deprotonated pores, suggesting that different methods of $\mathrm{SiO}$ pore preparation which can produce different interior surface pore structures are worth exploring in order to maximize the energy conversion. The advantage of deprotonation is not observed in the nonpolarizable model since $\mathrm{Cl}^{-}$ions represent the primary current carrier in these systems.

If we denote the volume flow $d V / d t$ by $Q$, the phenomenolog- ical Onsager relations for $Q$ and electric current $I$ can be written as

$$
Q=L_{11} \nabla P+L_{12} E
$$

and

$$
I=L_{21} \nabla P+L_{22} E .
$$

Within the linear response regime, coefficients $L_{i j}$ remain approximately constant and the optimal mechanical-to-electric energy conversion $\varepsilon \simeq L_{i \neq j}^{2} / 4 L_{11} L_{22}[56$. Using simulated coefficients $L_{21}$, the above expression for the conversion efficiency agrees with direct calculation of $P_{e l} / P_{\text {mech }}$ shown in Table 5, Unlike $L_{21}$, the EOF coefficient $L_{12}$ shows considerable dependence on $\nabla P$ leading to deviations from the Onsager's reciprocity principle. Its use in the linear response approximation would hence overestimate $\varepsilon$ compared to the actually observed energy conversion.

\section{Concluding remarks}

The exaggerated binding times and overall binding probabilities for ions, in particular cations, observed in our simulations using fixed-charge models strongly suggest that polarizable models may be required to properly describe the molecular interactions at the interface between the solution and the silica surface. Somewhat surprisingly, the long binding times are still seen in simulations combining a polarizable ion model with SPC/E water, demonstrating that the water model must also be polarizable to ensure correct results. While both the nonpolarizable and polarizable models are parameterized to match the correct hydration free energy in the bulk phase, the nonpolarizable model of water appears to underestimate the penalty of partial dehydration of ions adjacent to the wall, leading to a significant increase in ion-silica binding propensity in this model.

Notwithstanding the improved results we obtained with polarizable models, it must be said that the polarizable Drude oscillator models we have used in this work have not been properly parameterized to specifically describe the ion-silica interactions. Further work on parameterizing the interactions between the ions and the 
Table 5 Efficiency of energy conversion

\begin{tabular}{|c|c|c|c|c|c|c|c|c|c|c|}
\hline Model & $N_{N a^{+}}$ & $\mathrm{N}_{\mathrm{Cl} l^{-}}$ & $A_{g} / A_{1}$ & $\Delta N_{i} v_{i, E=0}$ & $d \Delta v / d E$ & $I_{\max }$ & $E_{o}$ & $P_{e l}$ & $P_{\text {mech }}$ & $\varepsilon$ \\
\hline & & & & $\mathrm{m} \mathrm{s}^{-1}$ & $(\mathrm{Vs})^{-1}$ & $\mathrm{nA}$ & $\mathrm{V} \mathrm{nm}^{-1}$ & $\mathrm{nW}$ & $\mathrm{nW}$ & $\%$ \\
\hline \multicolumn{11}{|l|}{ Protonated } \\
\hline \multirow[t]{4}{*}{$\overline{P O L_{A L L}}$} & 6 & 6 & 2.0 & 7.0 & 88 & 1.66 & 0.08 & 0.135 & 63 & 0.21 \\
\hline & 6 & 6 & 3.0 & 14.0 & 156 & 3.32 & 0.09 & 0.303 & 187 & 0.16 \\
\hline & 12 & 12 & 2.0 & 6.0 & 100 & 1.42 & 0.06 & 0.086 & 59 & 0.15 \\
\hline & 12 & 12 & 3.0 & 9.5 & 158 & 2.25 & 0.06 & 0.137 & 193 & 0.075 \\
\hline \multirow[t]{2}{*}{$N O P O L_{A L L}$} & 6 & 6 & 1.0 & -2.0 & 13 & -0.47 & -0.15 & 0.071 & 8 & 0.89 \\
\hline & 6 & 6 & 2.0 & -5.0 & 33 & -1.19 & -0.15 & 0.18 & 43 & 0.42 \\
\hline \multicolumn{11}{|c|}{ Deprotonated } \\
\hline \multirow[t]{4}{*}{$\overline{P O L_{A L L}}$} & 12 & 6 & 2.0 & 16.5 & 66 & 3.9 & 0.25 & 0.99 & 56 & 1.8 \\
\hline & 12 & 6 & 3.0 & 40 & 182 & 9.48 & 0.22 & 2.1 & 186 & 1.13 \\
\hline & 48 & 6 & 2.0 & 13 & 130 & 3.08 & 0.1 & 0.31 & 31 & 1.0 \\
\hline & 48 & 6 & 3.0 & 31 & 163 & 7.35 & 0.19 & 1.41 & 95 & 1.5 \\
\hline \multirow{2}{*}{$N O P O L_{A L L}$} & 12 & 6 & 1.0 & -2.2 & 24 & -0.52 & -0.12 & 0.063 & 8.8 & 0.3 \\
\hline & 12 & 6 & 2.0 & -7 & 58 & -1.66 & -0.12 & 0.202 & 49 & 0.35 \\
\hline
\end{tabular}

Table 6 Phenomenological coefficients

\begin{tabular}{|c|c|c|c|c|c|c|c|}
\hline$\overline{\text { Model }}$ & $\mathrm{N}_{\mathrm{Na}}$ & $\mathrm{N}_{\mathrm{Cl}^{-}}$ & $\overline{A_{g} / A_{1}}$ & $L_{11}$ & $L_{12}$ & $L_{22}$ & $L_{21}$ \\
\hline & & & & $\mathrm{m}^{3} \mathrm{~s} \mathrm{~kg}^{-1}$ & $m^{4}(\mathrm{Vs})^{-1}$ & $\mathrm{~A} \mathrm{~m} \mathrm{~V}^{-1}$ & $\mathrm{~A} \mathrm{~m}^{4} \mathrm{~J}^{-1}$ \\
\hline \multicolumn{8}{|l|}{ Protonated } \\
\hline \multirow[t]{4}{*}{$\overline{P O L_{A L L}}$} & 6 & 6 & 2.0 & $1.8 \times 10^{-34}$ & $8 \times 10^{-27}$ & $2.1 \times 10^{-17}$ & $5.4 \times 10^{-27}$ \\
\hline & 6 & 6 & 3.0 & $2.4 \times 10^{-34}$ & $8.5 \times 10^{-27}$ & $3.7 \times 10^{-17}$ & $7.2 \times 10^{-27}$ \\
\hline & 12 & 12 & 2.0 & $1.6 \times 10^{-34}$ & $6.3 \times 10^{-27}$ & $2.4 \times 10^{-17}$ & $4.6 \times 10^{-27}$ \\
\hline & 12 & 12 & 3.0 & $2.3 \times 10^{-34}$ & $14.5 \times 10^{-27}$ & $3.8 \times 10^{-17}$ & $4.9 \times 10^{-27}$ \\
\hline \multirow[t]{2}{*}{$N O P O L_{A L L}$} & 6 & 6 & 1.0 & $8.8 \times 10^{-35}$ & & $3.1 \times 10^{-18}$ & $3.1 \times 10^{-27}$ \\
\hline & 6 & 6 & 2.0 & $1.2 \times 10^{-34}$ & & $7.9 \times 10^{-18}$ & $3.9 \times 10^{-27}$ \\
\hline \multicolumn{8}{|c|}{ Deprotonated } \\
\hline \multirow[t]{4}{*}{$\overline{P O L_{A L L}}$} & 12 & 6 & 2.0 & $1.54 \times 10^{-34}$ & $2.9 \times 10^{-26}$ & $1.56 \times 10^{-17}$ & $1.27 \times 10^{-26}$ \\
\hline & 12 & 6 & 3.0 & $2.24 \times 10^{-34}$ & $6.6 \times 10^{-26}$ & $4.3 \times 10^{-17}$ & $2.05 \times 10^{-26}$ \\
\hline & 48 & 6 & 2.0 & $8.5 \times 10^{-35}$ & $3.1 \times 10^{-26}$ & $3.1 \times 10^{-17}$ & $1.0 \times 10^{-26}$ \\
\hline & 48 & 6 & 3.0 & $1.16 \times 10^{-34}$ & $1.0 \times 10^{-26}$ & $3.9 \times 10^{-17}$ & $1.59 \times 10^{-26}$ \\
\hline \multirow[t]{2}{*}{$N O P O L_{A L L}$} & 12 & 6 & 1.0 & $9.8 \times 10^{-35}$ & & $4.3 \times 10^{-18}$ & $3.4 \times 10^{-27}$ \\
\hline & 12 & 6 & 2.0 & $1.36 \times 10^{-34}$ & & $1.38 \times 10^{-17}$ & $5.4 \times 10^{-27}$ \\
\hline
\end{tabular}



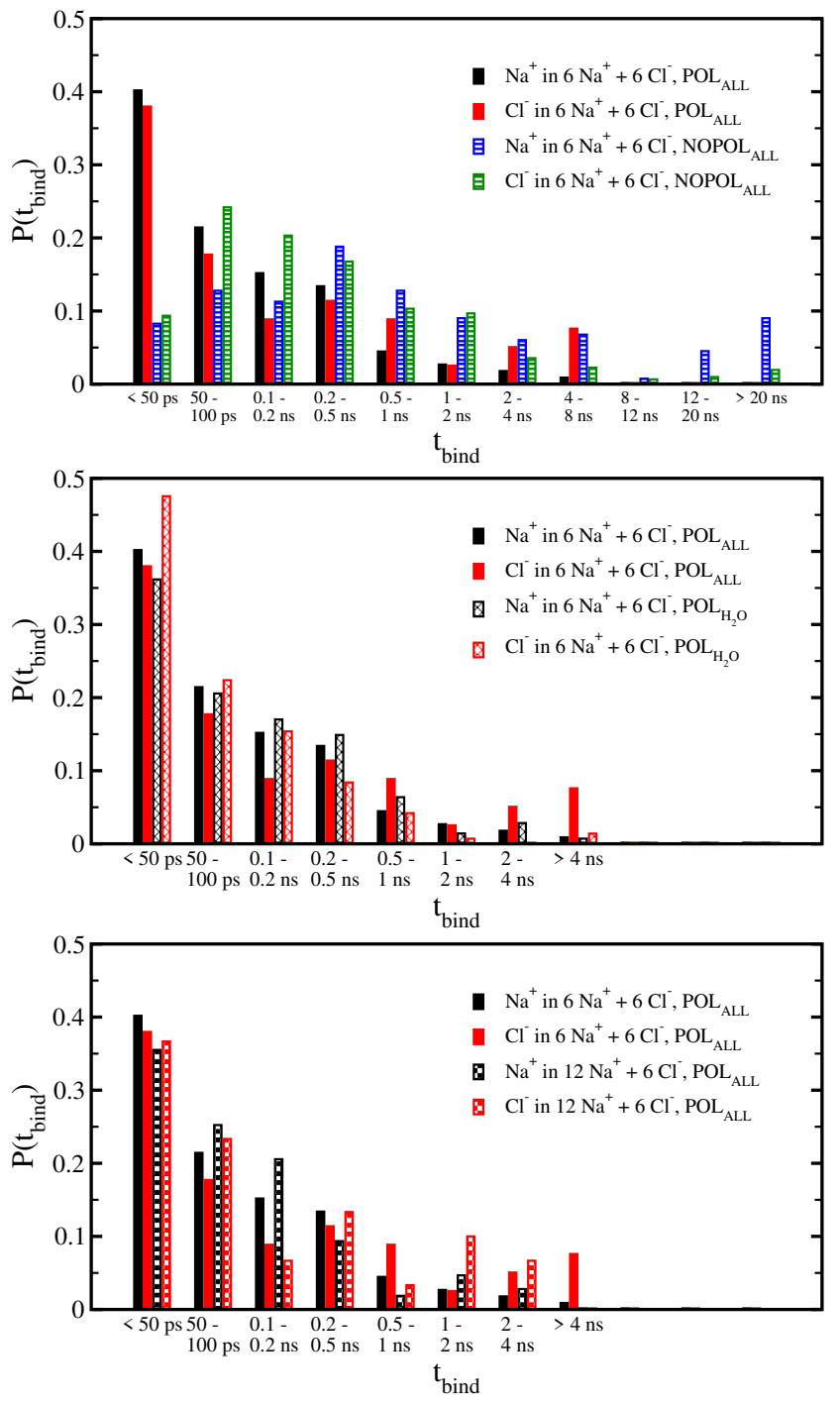

Fig. 5 Histogram of binding times for individual ion binding events, comparing results in the $P O L_{A L L}$ versus $N O P O L_{A L L}$ systems (top), the $P O L_{A L L}$ versus $\mathrm{POL}_{\mathrm{H}_{2} \mathrm{O}}$ systems (middle), and the $P O L_{A L L}$ system with fully protonated walls and $6 \mathrm{Na}^{+}$ions versus the system with partially deprotonated walls and $12 \mathrm{Na}^{+}$ions (bottom). These data are all for $A_{g} / A_{1}=2.0$ and $E_{z}=0$.

silica surface should be done. One approach to this would be to adapt recent advances in refining the description of specific interactions between ions and proteins for use with Drude oscillator models to the silica interface 57 .

Various deprotonation schemes have been proposed in previous works. For example, it has been argued that adding additional negative charge to deprotonated oxygens to counter the loss of each hydrogen may be more compatible with the CLAYFF force field 36 37. Use of this scheme would require the addition of approximately one $\mathrm{Na}^{+}$per deprotonated site, a factor of two increase over the approach we have used. There would be less deprotonated sites available for binding each $\mathrm{Na}^{+}$, but each site would bind much more strongly. Further work could examine how the ion binding is affected by different deprotonation strategies. It would also be worthwhile to be able to capture the correct (de)protonation chemistry at the silica interface with a model capable of describing the breaking and making of the $\mathrm{OH}$ bonds. One intriguing possibility which might be able to describe the physics without the significant challenge of running long AIMD simulations would be the ReaxFF family of force fields, variants of which have been developed to describe the silica-water interface ${ }^{58}$ as well as $\mathrm{Na}^{+}$and $\mathrm{Cl}^{-}$-water interactions ${ }^{59}$. Recent simulation results using ReaxFF to study aqueous $\mathrm{NaCl}$ interactions with silica generally agree with our results for polarizable force fields 51 . However, as with the Drude oscillator models it is likely some reparameterization could be required in order to accurately describe the ion-silica interactions 60 .

Comparison with recent results showing that $\mathrm{Na}^{+}$binding to phospholipid bilayers is overestimated by non-polarizable force fields ${ }^{52153}$, along with the large body of work showing that large anions should be modelled with polarizable force fields to properly describe their interfacial interactions $s^{2324}$ strongly suggests that some explicit treatment of polarizability should be included in future improvements in descriptions of the interaction with silica of both cations and anions, as well as with water.

It should be noted that in experiments salt concentration in the nanopores can deviate from that of the bulk solution ${ }^{61}$ although the extent of exclusion is hard to quantify in simulations, even at equilibrium conditions 1920 . One can hence expect not only considerable electrostatic but also detectable composition gradients at nanopore ends present in practical devices ${ }^{62}$. Such gradients have been known to play an important role in determining the efficiency of electrokinetic conversion ${ }^{63}$. Moreover, significant reduction of back conductance can be achieved due to turbulent pore exhaust conditions ${ }^{25}$. Inclusion of static and dynamic endeffects is therefore a worthwhile extension for our future studies, among many others we have mentioned, but is outside the scope of the present analysis.

Our measured power conversion efficiencies do not exceed $2 \%$, a number several times less than the efficiencies ca. $10 \%$ measured by some experiments on fluid flow through small pores $25 \mid 26$. These experiments study pores with diameters in the micrometer range, and millimolar ion concentrations, and so our nanoscale molecular simulations cannot be expected to match these experiments too closely. These differences notwithstanding, our finding that deprotonation of the pore surface can lead to an order of magnitude improvement in efficiency might indicate that new experiments to generate electric power from flowing electrolyte should consider the impact of different methods for preparation of silica pores with different surface characteristics in order to maximize the efficiency. Our future work will address such modifications, in addition to potential improvements achievable by selection of ions of bigger size $\frac{64}{4}$, the use of nanojet exhaust from the device to minimize back-current $\frac{65}{6}$, and introduction of slippage-conducive coating to reduce dissipation and hence the mechanical work 4765 . Parallel methodological and force fieldadvances will be essential for successful in silico predictions of these effects.

\section{Conflicts of interest}

There are no conficts to declare. 

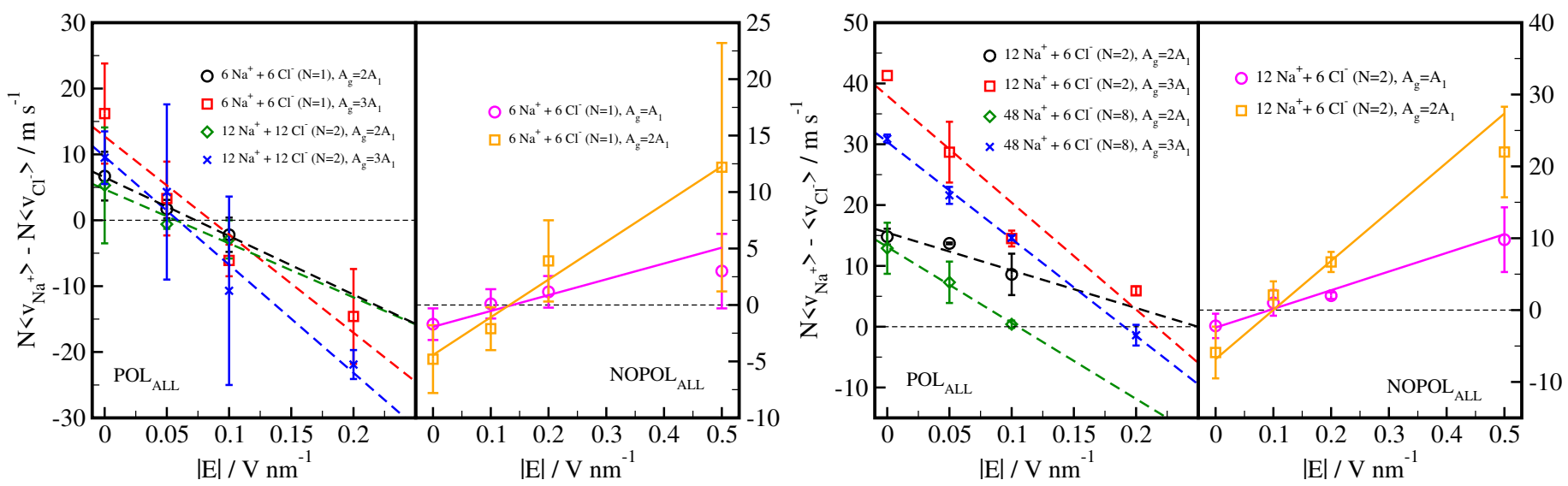

Fig. 6 Plots of the average difference between positive and negative ion flow velocities as a function of applied field strength. Factors $N=N_{\mathrm{Na}^{+}} / 6$ allow comparisons between different concentrations of each ion type. Left: Fully protonated neutral pore. Right: Partially deprotonated negatively charged pore. Lines are linear fits to the data for each system.

\section{Acknowledgements}

CDD and NMC thank the Natural Sciences and Engineering Research Council (NSERC) of Canada for support. CDD, DB, and AL acknowledge support from the Office of Basic Energy Sciences, Chemical Sciences, Geosciences, and Biosciences Division of the U.S. Department of Energy (Grant No. DE-SC 0004406). This research used computing resources of Compute Canada and NOTUR account nn-2920k, the National Energy Research Scientific Computing Center (NERSC) supported by the U.S. Department of Energy (Contract No. DEAC02-05CH11231), and the Extreme Science and Engineering Discovery Environment (XSEDE) supported by the National Science Foundation (Grant No. OCI1053575).

\section{Notes and references}

1 F. L. Mantia, M. Pasta, H. D. Deshazer, B. E. Logan and Y. Cui, Nano Lett., 2011, 11, 1810-1813.

2 O. S. Burheim, F. Liu, B. B. Sales, O. Schaetzle, C. J. N. Buisman and H. V. M. Hamelers, J. Phys. Chem. C, 2012, 116, 19203-19210.

3 K. Leung, S. B. Rempe and C. D. Lorenz, Phys. Rev. Lett., 2006, 96, 095504.

4 K. Leung, J. Am. Chem. Soc., 2008, 130, 1808-1809.

5 D. Argyris, D. R. Cole and A. Striolo, ACS Nano, 2010, 4, 2035-2042.

6 P. E. Videla, J. Sala, J. Martí, E. Guàrdia and D. Laria, J. Chem. Phys., 2011, 135, 104503.

7 T. A. Ho, D. Argyris, D. R. Cole and A. Striolo, Langmuir, 2012, 28, 1256-1266.

8 I. C. Bourg and C. I. Steefel, J. Phys. Chem. C, 2012, 116, 11556-11564.

9 A. Moshfegh and A. Jabbarzadeh, Microfluid. Nanofluid., 2016, 20, 67.

10 R. Hartkamp, B. Siboulet, J.-F. Dufrêche and B. Coasne, Phys. Chem. Chem. Phys., 2015, 17, 24683.

11 C. D. Lorenz, P. S. Crozier, J. A. Anderson and A. Travesset, J. Phys. Chem. C., 2008, 112, 10222-10232.
12 N. R. Haria and C. D. Lorenz, Phys. Chem. Chem. Phys., 2012, 14, 5935-5944.

13 N. R. Haria and C. D. Lorenz, J. Phys. Chem. C, 2015, 119, 12298-12311.

14 H. A. Zambrano, M. Pinti, A. T. Conlisk and S. Prakash, Microfluid. Nanofluid., 2012, 13, 735-747.

15 A. Boţan, V. Marry, B. Rotenberg, P. Turq and B. Noetinger, J. Phys. Chem. C, 2013, 117, 978-985.

16 B. Rotenberg and I. Pagonabarraga, Molec. Phys., 2013, 111, 827-842.

17 H. J. C. Berendsen, J. R. Grigera and T. P. Straatsma, J. Phys. Chem., 1987, 91, 6269-6271.

18 I. S. Joung and T. E. Cheatham, III, J. Phys. Chem. B, 2008, 112, 9020-9041.

19 F. Moucka, D. Bratko and A. Luzar, J. Chem. Phys., 2015, 142, 20416-20425.

20 F. Moucka, D. Bratko and A. Luzar, J. Phys. Chem. C, 2015, 142, 124705.

21 P. Drude, The Theory of Optics, Longmans, Green, New York, 1902.

22 G. Lamoureux and B. Roux, J. Chem. Phys., 2003, 119, 30253039.

23 P. B. Petersen and R. J. Saykally, Ann. Rev. Phys. Chem., 2006, 57, 333-364.

24 C. Caleman, J. S. Hub, P. J. van Maaren and D. van der Spoel, Proc. Nat. Acad. Sci., 2011, 108, 6838-6842.

25 A. M. Duffin and R. J. Saykally, J. Phys. Chem. C, 2008, 112, 17018-17022.

26 D. N. Kelly, R. K. Lam, A. M. Duffin and R. J. Saykally, J. Phys. Chem. C, 2013, 117, 12702-12706.

27 G. Lamoureux, A. D. MacKerell Jr. and B. Roux, J. Chem. Phys., 2003, 119, 5185-5197.

28 G. Lamoureux, E. Harder, I. V. Vorobyov, B. Roux and A. D. MacKerell Jr., Chem. Phys. Lett, 2006, 418, 245-249.

29 H. Yu, T. W. Whitfield, E. Harder, G. Lamoureux, I. Vorobyov, V. M. Anisimov, J. A. D. MacKerell and B. Roux, J. Chem. Theory Comput., 2010, 6, 774-786. 
30 C. D. Daub and N. M. Cann, J. Phys. Chem. A, 2012, 116, 10488-10495.

31 R. T. Cygan, J.-J. Liang and A. G. Kalinichev, J. Phys. Chem. B, 2004, 108, 1255-1266.

32 L. T. Zhuravlev, Coll. Surf. A: Phys. Chem. Eng. Aspects, 2000, 173, 1-38.

33 E. R. Cruz-Chu, A. Aksimentiev and K. Schulten, J. Phys. Chem. B, 2006, 110, 21497-21508.

34 S. Kerisit and C. Liu, Environ. Sci. Technol., 2009, 43, 777782.

35 R. Renou, A. Szymczyk and A. Ghoufi, J. Chem. Phys., 2014, 140, 044704.

36 L. N. Lammers, I. C. Bourg, M. Okamura, K. Kolluri, G. Sposito and M. Machida, J. Colloid Interface Sci., 2017, 490, 608-620.

37 M. Collin, S. Gin, B. Dazas, T. Mahadevan, J. Du and I. C. Bourg, J. Phys. Chem. C, 2018, 122, 17764-17776.

38 M. E. Suk and N. R. Aluru, RSC Adv., 2013, 3, 9365-9372.

39 L. Wang, R. S. Dumont and J. M. Dickson, Mol. Phys., 2017, 115, 981-990.

40 R. Khare, J. de Pablo and A. Yethiraj, J. Chem. Phys., 1997, 49, 2589-2596.

41 J. A. Thomas, A. J. H. McGaughey and O. Kuter-Arnebeck, Int. J. Therm. Sci., 2010, 49, 281-289.

42 S. De Luca, B. D. Todd, J. S. Hansen and P. J. Daivis, J. Chem. Phys., 2014, 140, 054502.

43 M. Thomas and B. Corry, Microfluid. Nanofluid., 2015, 18, 41-47.

44 M. P. Allen and D. J. Tildesley, Computer simulation of liquids, Clarendon Press, Oxford, 2017.

45 M. Neumann and O. Steinhauser, Molec. Phys., 1980, 39, 437454.

46 I.-C. Yeh and M. L. Berkowitz, J. Chem. Phys., 1999, 111, 3155.

47 D. J. Bonthuis, K. F. Rinne, K. Falk, C. N. Kaplan, D. Horinek, A. N. Berker, L. Bocquet and R. R. Netz, J. Phys.: Condens. Matter, 2011, 23, 184110.

48 C. Sendner, D. Horinek, L. Bocquet and R. R. Netz, Langmuir, 2009, 25, 10768-10781.

49 M. A. Gonzalez and J. L. F. Abascal, J. Chem. Phys., 2010, 132, 096101.

50 P. M. Dove and C. M. Craven, Geochim. Cosmochim. Ac., 2005, 69, 4963-4970.

51 J. M. Rimsza, R. E. Jones and L. J. Criscenti, J. Colloid Interface Sci., 2018, 516, 128-137.

52 A. Catte, M. Girych, M. Javanainen, C. Loison, J. Melcr, M. S. Miettinen, L. Monticelli, J. Määttä, V. S. Oganesyan, O. H. S. Ollila, J. Tynkkynen and S. Vilov, Phys. Chem. Chem. Phys., 2016, 18, 32560-32569.

53 J. Melcr, H. Martinez-Seara, R. Nencini, J. Kolafa, P. Jungwirth and O. H. S. Ollila, J. Phys. Chem. B, 2018, 122, 45464557.

54 I. C. Bourg, S. S. Lee, P. Fenter and C. Tournassat, J. Phys. Chem. C, 2017, 121, 9402-9412.

55 L. X. Dang and D. E. Smith, J. Chem. Phys., 1995, 102, 3483-
3484.

56 F. H. J. van der Heyden, D. J. Bonthuis, D. Stein, C. Meyer and C. Dekker, Nano Lett., 2006, 6, 2232-2237.

57 H. Li, V. Ngo, M. C. D. Silva, D. R. Salahub, K. Callahan, B. Roux and S. Y. Noskov, J. Phys. Chem. B, 2015, 119, 94019416.

58 J. C. Fogarty, H. A. Aktulga, A. Y. Grama, A. C. T. van Duin and S. A. Pandit, J. Chem. Phys., 2010, 132, 174704.

59 S.-Y. Kim and A. C. T. van Duin, J. Mater. Res., 2013, 28, 513520.

60 P. Li and K. M. Merz Jr., Chem. Rev., 2017, 117, 1564-1686.

61 S. Kuyucak, O. S. Andersen and S.-H. Chung, Rep. Prog. Phys., 2001, 64, 1427-1472.

62 R. B. Schoch, J. Han and P. Renaud, Rev. Mod. Phys., 2008, 80, 839.

63 Y. Zhang, Y. He, M. Tsutsui, X. S. Miao and M. Taniguchi, Sci. Rep., 2017, 7, 46661.

64 C. Bakli and S. Chakraborty, Electrophoresis, 2015, 36, 675681.

65 N. Schwierz, R. K. Lam, Z. Gamlieli, J. J. Tills, A. Leung, P. L. Geissler and R. J. Saykally, J. Phys. Chem. C, 2016, 120, 14513-14521. 


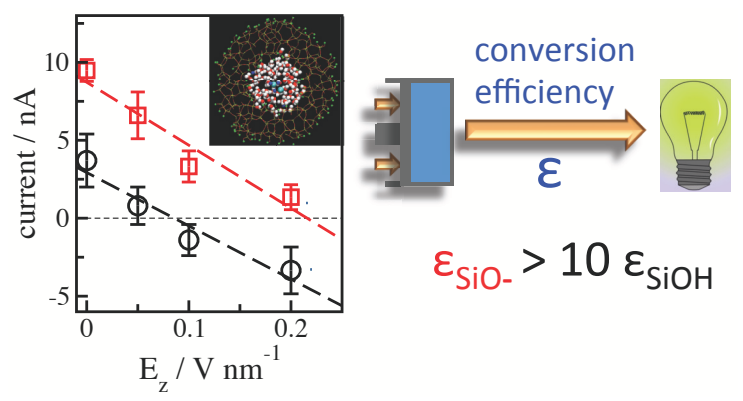

Fig. 7 TOC GRAPHIC. Electrokinetic energy conversion upon a pressure-driven flow of electrolyte in a silica nanopore shows a tenfold increase upon partial nanopore deprotonation. 